\title{
Organic molecules in saturnian E-ring particles. Probing subsurface oceans of Enceladus?
}

\author{
Frank Postberg ${ }^{1}$, S. Kempf ${ }^{2}$, R. Srama ${ }^{3}$, E. Grün ${ }^{3}$, J. K. Hillier ${ }^{4}$, \\ S. F. Green ${ }^{4}$, and N. McBride ${ }^{4}$ \\ ${ }^{1}$ MPI für Kernphysik, Heidelberg, Germany \\ email: Frank.Postberg@mpi-hd.mpg.de \\ ${ }^{2}$ MPI für Kernphysik, Heidelberg, Germany and Institut für Geophysik und extraterrestrische \\ Physik Universität Braunschweig, Germany \\ ${ }^{3}$ MPI für Kernphysik, Heidelberg, Germany \\ ${ }^{4}$ Planetary and Space Science Research Institute, The Open University, Milton Keynes, U. K.
}

\begin{abstract}
The population of Saturn's outermost tenuous E-ring is dominated by tiny water ice particles, some of which contain organic or mineral impurities. Active cryo-volcanism on the moon Enceladus, embedded in the E-ring, has been known to be a major source of particles replenishing the ring since late 2005. Therefore, particles in the vicinity of Enceladus provide crucial information about the dynamic and chemical processes occurring far below the moon's icy surface.

We present a compositional analysis of thousands of impact ionisation mass spectra of Saturn's E-ring particles, with sizes predominantly below $1 \mu \mathrm{m}$, detected by the Cosmic Dust Analyser onboard the Cassini spacecraft. Our findings imply that organic compounds are a significant component of icy particles ejected by Enceladus plumes. Our in situ measurements are supported by detections of other Cassini instruments. They hint at a dynamic interaction of a hot rocky core with liquid water below the icy surface, where the organic molecules are generated. Further insights are expected from two close Enceladus flybys to be performed by Cassini in 2008. Then, for the first time, we will obtain spectra of freshly ejected particles at the traversals through the cryo-volcanic plumes.
\end{abstract}

\section{Discussion}

Mumma: I gather that you did not detect any nitrogen in your samples. Is that correct?

PostberG: That is correct, yes.

Mumma: That's surprising considering the strength of the cyanogen bond. You have $600 \mathrm{~K}$ temperatures in the interior of Saturn processing other materials. I can't imagine you wouldn't make triple bonded $\mathrm{CN}$ and then get some kind of nitriles.

POstberg: Keep in mind that we are analyzing this in solid phase and we are not very sensitive to nitrogen. We are only seeing the cations, and nitrogen doesn't like to form any cations. So we cannot exclude the possibility that nitrogen could be part of the particles, but we don't detect it.

ZIURYS: How unique is your interpretation of your time-of-flight mass spectral data that everything is entirely due to water and hydrocarbons? Could you have oxygen and nitrogen mixed in? 
PostberG: I wouldn't say that there is only hydrogen and carbon. There could be other functional groups as well, but so far our analysis is just not precise enough. With the upcoming plume crossings, we hope to get spectra of freshly ejected plume particles of different impact speeds, and then we probably will be able to work out a more precise picture.

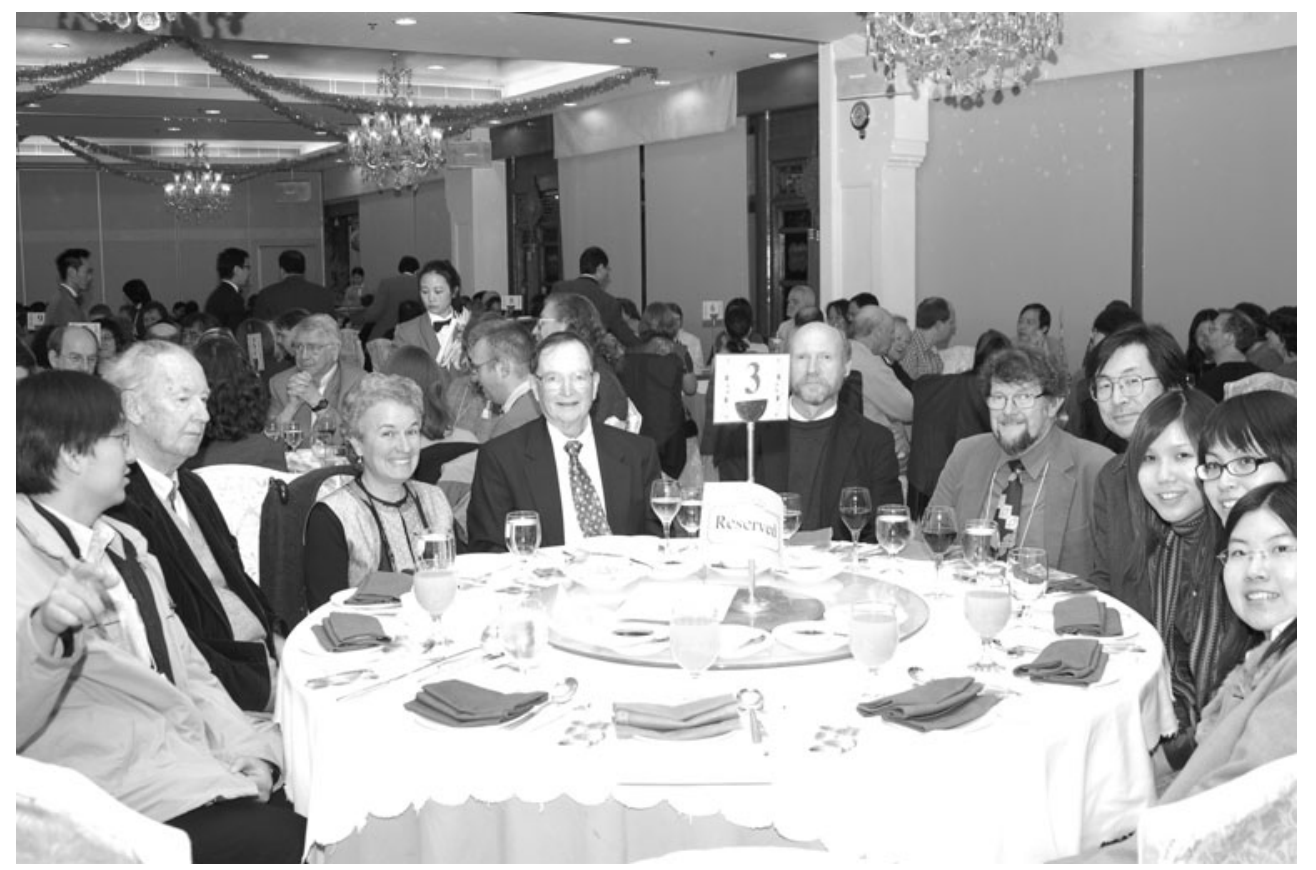

From left to right: Henry Chan, Cliff Matthews, Sandra Matthews, Bill Irvine, Hans Olofsson, Åke Hjalmarson, Masatoshi Ohishi, Daisy Mak, Gloria Cheung, Selina Chong. 